\title{
Celebratory message from the Editor-in-Chief on the 50th anniversary of the Journal of Gastroenterology
}

\author{
Akira Andoh
}

Published online: 24 February 2015

(C) Springer Japan 2015

In 2015 we celebrate the 50th anniversary of publication of the Journal of Gastroenterology, the official journal of the Japanese Society of Gastroenterology.

Two years ago, Professor Mamoru Watanabe, director of the Japanese Society of Gastroenterology, called me to consider getting to Editor-in-Chief of the Journal of Gastroenterology. Although it seemed to surpass my ability, I felt a bit overwhelmed at the beginning. With feeling worry, I began my editorship in April of 2013, and in 2 years it was completed. Now, I am glad I did it. For this mission, I really appreciate the sincere and continuous support of Prof. Mamoru Watanabe, Prof. Naoya Sakamoto vice Editor-in Chief, and all of the associate editors who are great leaders of gastroenterology in Japan and large secretariats of the society. In addition, this success was actually dependent on enthusiastic contribution of the peer reviewers all over the world.

In these 2 years, we have made some challenges to make the journal more attractive. At first, we changed the cover and the style of the first page of each article. The cover of the Journal of Gastroenterology was entirely painted by dark blue and slightly business-like look of previous years. We introduced pale blue in the lower half part of the cover with an illustration or picture from the included articles. A blue mark line and the symbol of the society were drawn on the top of the first page of each article. Through these changes, we aimed to call the Journal of Gastroenterology "Blue Journal" for the nickname. Authors sometimes publish the figures as a blackand-white version, although they submit color figures for

\footnotetext{
A. Andoh $(\bowtie)$

Department of Medicine, Shiga University of Medical Science,

Seta-Tukinowa, Otsu 520-2192, Japan

e-mail: andoh@belle.shiga-med.ac.jp
}

the review process. So, we extremely reduced the charge for color figures, and now all color figures on submission are published in color in the journal. In the future, to give the journal a more contemporary look, visual illustrations may be helpful in making complex processes easily understandable. Indeed, Gastroenterology, the official journal of the American Gastroenterological Association, introduced a medical illustrator to the editorial staff to make cover illustrations and to redrew all author-submitted line art for increased consistency of style. In addition, the entire peer-review process is getting much faster and the first decision for a submitted paper is completed within 16 days. Therefore, the time from acceptance to publication is getting shorter.

As a second challenge, we aimed to increase the journal status as an international journal. For this purpose, we made efforts to increase the impact factor. The impact factor has been used as a quantitative measure of the status of a journal. An increase in the impact factor attracts submissions and gives a signal of the quality of the published articles. At first, we terminated publication of case reports on August 2013, since the low citation rates of the case reports was affecting the impact factor at that time. Through tremendous efforts of the editorial team including associate editors and peer reviewers to make strict decisions, the impact factor of the Journal of Gastroenterology has progressively increased, reaching a value of 4.020 in 2013 (Fig. 1). It was ranked 15th of 75 journals in the gastroenterology. The total number of submissions increased from approximately 250 at the beginning of the year 2000 to approximately 1,200 in 2013. Citation of articles from Europe and Pacific regions is increasing (Fig. 2). In contrast, strict peer-review policy resulted in the acceptance rate dropping to a low of $12.7 \%$ in 2013. There is a discussion whether the Journal of 
Fig. 1 Impact factor of the recent 10 years (Source: Journal Citation Reports)
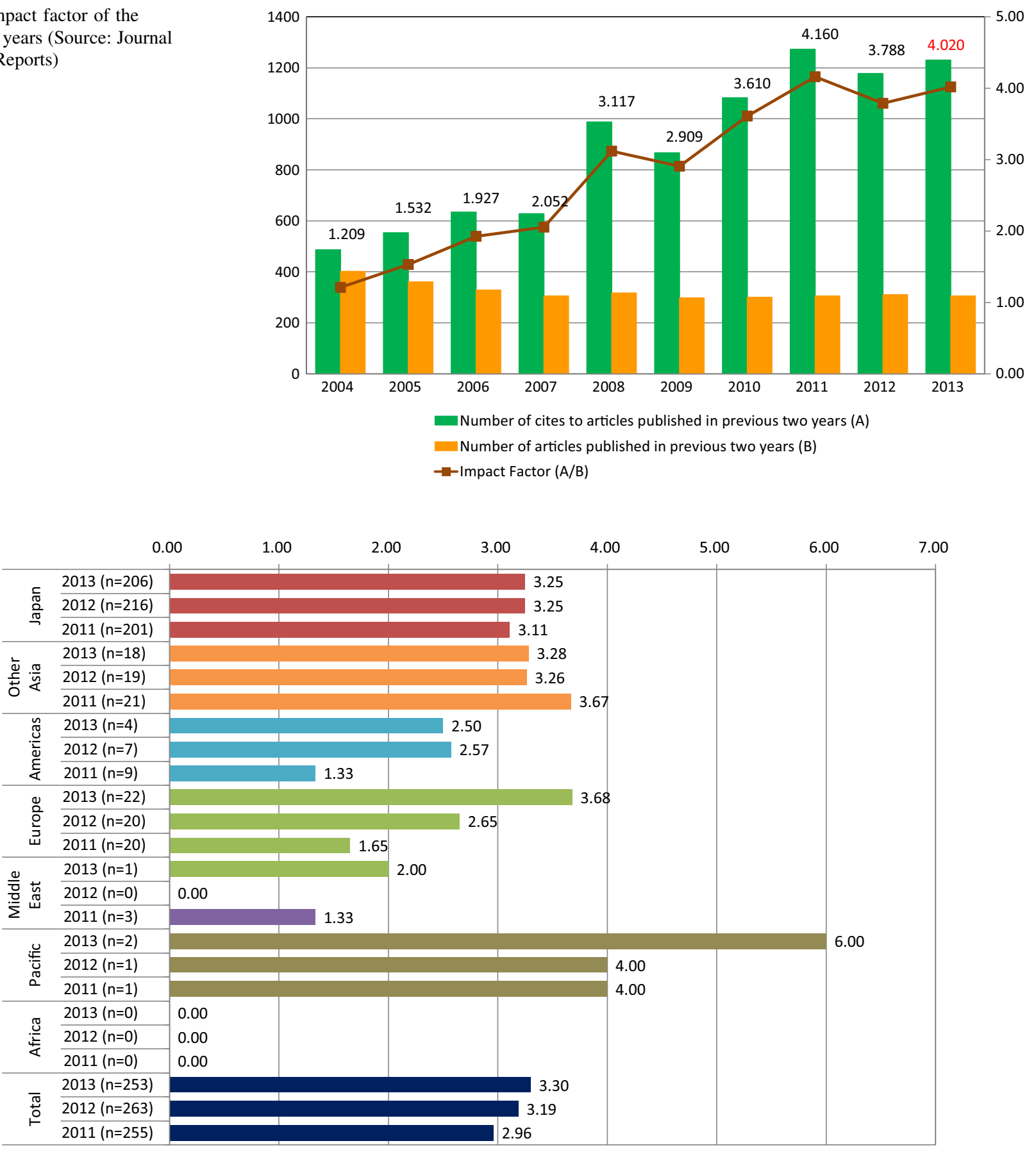

Fig. 2 Average citation of original articles globally (Source: Web of Science, as of 2014)

Gastroenterology should publish the best research in gastroenterology or publish all materials or papers that may be of interest to the membership of the society. Concerning this point, I hope the member to put up with low acceptance rate only for a while. I think that it is a transition period of the journal to be recognized as mature international journal.

The submission of international papers continues to increase and reached 780 (65\% of total submission) in 2013.
However, the acceptance rate of international papers is only $6 \%$, in contrast to approximately $25 \%$ for domestic papers. The net result is that international papers were approximately $30 \%$ of original papers published in the journal in 2013. This is not the result of biased reviews, but due to slightly low originality or quality of international papers. How will the journal continue to be competitive in attracting the best original papers of the world? I think that one of the ways to attract high-quality papers is to provide 
timely, supportive, and high-quality review papers. For this, the Journal of Gastroenterology is planning to invite international experts as international editors.

Collection of high-quality review papers has another importance. The main objects of the Journal of Gastroenterology over the years has been the reporting and documentation of original and high-impact findings of the fields of gastroenterology from both the laboratory bench and clinical bed side. However, according to the accumulation of such primary information, making a connection between the many disconnected fields of the original reports is being required in order to give an overview of the crosstalk between many fields of science. For example, it is impossible to discuss the pathophysiology of inflammatory bowel disease without the recent advance of immunology and deep understanding of gut microbiota. The pathophysiology of non-alcoholic steatohepatitis is well known to be associated with diabetes mellitus or abnormality of energy metabolism. To achieve this task, collection of good review papers of various fields is important. We have invited many well-known specialists from various fields not only in gastroenterology but also other topic fields to give a chance to connect many disconnected fields of the science. It will take a little more time to know whether our mission was really successful or not.

There are some issues for the Journal of Gastroenterology to be considered. For accessibility and appeal of the journal, Prof. Sugano, the previous president of the Japanese Society of Gastroenterology, mentioned that providing commentaries on the articles by editorial members is effective in highlighting the new findings of the journal [1]. Some journals also utilize social network services as providing tool of journal information to increase the awareness of their articles. Although such efforts have not been tried in the Journal of Gastroenterology, realization in near future is our task to increase attractively of the journal. We should also consider how to freely access the articles of publicly funded research. It is well known that all National Institutes of Health (NIH)-funded papers must be deposited into PubMed for open access after 12 months of publication. The Journal of Gastroenterology is already ready for such a condition. As another approach, the journal keeps in mind to become open-access electronic journals, since print journals are expensive and difficult to read, store, and access. Finally, we are required to consider involving professional writers and illustrators for the enhancement of the accepted paper with simple and understandable concepts.

Basic and translational research is constantly developing and the Journal of Gastroenterology is facing an environment of new era, generated by high-technology platforms to analyze gene expression and proteomic and metabolomic patterns. In response to such evolution, the journal must enhance a new web-based approach to review and present an enormous amount of data. I hope that through such an approach the Journal of Gastroenterology gets to be much more flourished and stronger in many ways without delay.

\section{References}

1. Sugano K. Journal of Gastroenterology in the era of globalization. J Gastroenterol. 2015;50:1-3. 